\title{
ANÁLISE DA CONVECÇÃO RESOLVIDA EXPLICITAMENTE PELO MODELO BRAMS A PARTIR DA COMPARAÇÃO COM RADIÂNCIAS DE SATÉLITES.
}

\author{
RENATO GALANTE NEGRI, LUIZ AUGUSTO TOLEDO MACHADO, SAULO RIBEIRO DE FREITAS
}

\author{
Instituto Nacional de Pesquisas Espaciais, Centro de Previsão de Tempo e Estudos Climáticos, Cachoeira \\ Paulista, SP, Brasil,
} renato.galante@gmail.com, luiz.machado@cptec.inpe.br, saulo.freitas@cptec.inpe.br

Recebido Fevereiro de 2014 - Aceito Janeiro de 2015

\begin{abstract}
RESUMO
Este trabalho apresenta a análise da convecção resolvida explicitamente pelo modelo BRAMS, referentes ao projeto CHUVA, a partir da comparação com a convecção real presente nas imagens de satélites. Radiâncias em diferentes canais do infravermelho e micro-ondas, medidas pelos sensores GOES-imager e SSMI-S, foram simuladas pelo modelo de transferência radiativa RTTOV e comparadas às respectivas radiâncias medidas pelos sensores reais. Para tais simulações, os perfis atmosféricos provenientes do BRAMS foram utilizados como vetor estado da atmosfera. Devido à natureza caótica da atmosfera, principalmente na escala de nuvens, é inviável realizar a comparação entre as radiâncias simuladas e as medidas reais considerando a mesma posição geográfica. Portanto, as comparações foram feitas verificando se o modelo reproduz os aspectos gerais da convecção resolvida pelo modelo, independente da posição geográfica das nuvens. O projeto CHUVA realizou experimentos de campo em sete sítios com diferentes padrões climáticos, visando estudar os regimes de precipitação presentes no Brasil. A metodologia de análise apresentada foca apenas nas simulações referentes ao experimento Vale do Paraíba. Os resultados indicam que tal método de análise é viável para a análise das simulações dos demais experimentos de campo do projeto.
\end{abstract}

Palavras-chave: Modelo em escala de nuvens; avaliação; satélites meteorológico.

\begin{abstract}
ANALYSIS OF THE CONVECTION EXPLICITLY RESOLVED BY BRAMS USING SATELLITE RADIANCES

This work shows the analysis of the convection explicitly resolved by BRAMS model, done as part of the CHUVA project, where the resolved clouds and the ones present on real satellite images were compared. Radiances at infrared and microwave channels of GOES-Imager and SSMI-S were simulated using the radiative transfe rmodel RTTOV and then compared to its respective real images. The atmospheric profiles simulated by BRAMS were used as state vector to RTTOV. This comparison verified if the BRAMS was capable to reproduce the general behavior of the convection, despite erros in the geographic placement of individual clouds. For CHUVA project, seven field experiments over different locations of Brazil, dominated by distinct climate patterns, were done to study the various preciptation regimes over the country. The metodology presented here was applied only on the Vale do Paraiba experiment and the results shows that this metodology can be applied to the other field experiments of the CHUVA project.
\end{abstract}

Keywords: Cloud scale model, evaluation; meteorological satellites

\section{INTRODUÇÃO}

Com o constante aumento da capacidade computacional, tornou-se possível nos presentes dias simular o estado da atmosfera com resoluções espacial e temporal cada vez maiores. Os modelos numéricos sofreram melhorias nos últimos anos para serem executados com maior resolução espacial e para descrever explicitamente os processos das nuvens. Em muitos centros, modelos regionais com resolução espacial em torno de 12 até $4 \mathrm{~km}$ tem sido implementados operacionalmente, sendo previsto que nos próximos anos os modelos com resolução espacial de $1 \mathrm{~km}$ serão comuns (Lean et al., 2008). Muitos 
centros operacionais de previsão de tempo e clima estão voltando seus esforços no sentido de produzir previsões de curto prazo a partir de modelos numéricos capazes de resolver sistemas de nuvens individuais (comumente conhecidos pelo termo inglês cloud resolving models). Uma grande motivação para essa escolha é aumentar a precisão das previsões de eventos extremos como, por exemplo, situações de convecção severa, as quais podem causar diversos transtornos e prejuízos à sociedade como alagamentos, destruição de plantações e bens materiais. Diversos estudos mostram que resoluções espaciais maiores podem aumentar a capacidade de previsão dos modelos numéricos (Romero et al., 2001; Speer e Leslie 2002; Done et al., 2004). Ao aumentar a resolução horizontal de $100 \mathrm{~km}$ para $50 \mathrm{~km}$, algumas melhoras na simulação do ciclo diurno foram observadas por Ploshay e Lau (2010). Quando a resolução espacial é elevada para pelo menos $10 \mathrm{~km}$ torna-se possível resolver sistemas de nuvens e as interações existentes entre eles. Nessas resoluções a precipitação e a sua variabilidade podem ser simuladas de forma muito mais realista (Sato et al., 2009).

À medida que a resolução espacial dos modelos torna-se maior, possibilitando resolver nuvens explicitamente, surge a necessidade de avaliar quão realística é a representação da convecção produzida por tais modelos. Uma forma de realizar essa avaliação é por meio de imagens de satélites. Satélites geo-estacionários são uma alternativa interessante, pois possibilitam acompanhar o ciclo de vida da convecção presente na área imageada. Diversos autores estudaram o uso de imagens de satélites meteorológicos para avaliar modelos numéricos em escala de nuvens (Chaboureau et al. 2000; Ploshay e Lau, 2010). Negri et al. (2014) apresentam uma metodologia de análise de um modelo de previsão de tempo em escala de nuvens a partir da comparação entre radiâncias do infravermelho termal, simuladas utilizando perfis atmosféricos de tal modelo numérico e um modelo de transferência radiativa, e radiâncias de satélites geo-estacionários.

Atualmente, os processos físicos dentro das nuvens são um dos componentes mais desconhecidos dos sistemas de tempo e clima. Por exemplo, as nuvens quentes são responsáveis por grande parte da precipitação nos trópicos, principalmente nas regiões costeiras e esse tipo de nuvem é pouco estudado, não sendo considerado nas estimativas de precipitação por satélite. A descrição dos processos físicos que ocorrem nas nuvens através da utilização de parâmetros meteorológicos parametrizados em modelos numéricos deverá ser fortemente melhorada para descrever com precisão as características dos hidrometeoros, perfis de aquecimento latente, balanço radiativo, o entranhamento do ar e as correntes ascendentes e descendentes de ar no interior das nuvens. O projeto CHUVA (Cloud processes of the main precipitation systems in Brazil: A contribUtion to cloud resolVing modeling and to the GPM (GlobAl Precipitation Measurement)) visou realizar experimentos de campo em sete sítios com diferentes padrões climáticos com o intuito de estudar os regimes de precipitação presentes no Brasil. Esses experimentos utilizam diversos instrumentos como radar de dupla polarização, Lidar, radiômetro de micro-onda, disdrômetros, radiossondas e vários outros. Os objetivos principais do projeto são reduzir as incertezas na estimativa da precipitação e progredir no conhecimento dos processos das nuvens. O projeto CHUVA (Machado et al. 2014) focou na evolução da microfísica e do ciclo de vida das nuvens, o uso de diferentes algoritmos de estimação de precipitação, o desenvolvimento e formação de tempestades e relâmpagos, processos na camada limite, bem como de modelagem microfísica de nuvens.

Do ponto de vista da componente voltada à modelagem da microfísica de nuvens, este trabalho avaliou o uso da metodologia apresentada por Negri et al. (2014) para avaliação das simulações em escala de nuvens realizadas pelo modelo BRAMS como parte do projeto CHUVA, adaptando-a para o conjunto de dados disponíveis. Este trabalho apresenta os resultados preliminares das análises das simulações referentes ao projeto CHUVA, apresentando apenas a análise referente ao dia 28 de novembro de 2011. Os resultados obtidos indicaram algumas deficiências nas simulações e mostram que a metodologia pode ser aplicada com sucesso aos conjuntos de simulações dos diferentes experimentos de campo do projeto CHUVA.

\section{DADOS E METODOLOGIA}

Este estudo é baseado na metodologia descrita por Negri et al. (2014), em que o ciclo de vida da convecção e os aspectos macrofísicos das nuvens resolvidas pelo modelo numérico são avaliadas a partir da comparação com medidas de satélites meteorológicos. Nessa comparação, radiâncias dos sensores a bordo dos satélites em questão são simuladas através de um modelo de transferência radiativa, o qual utiliza como estado da atmosfera os perfis calculados pelo modelo numérico que se deseja avaliar, no caso, o BRAMS.

Neste estudo, radiâncias na porção do micro-ondas do espectro eletromagnético também foram utilizadas, enquanto que Negri et al. (2014) limitaram-se apenas ao infravermelho termal.

As seguintes observações meteorológicas e ferramentas computacionais foram utilizadas:

1. Rodadas BRAMS com resolução espacial de 1 x 1 $\mathrm{km}$ em domínios de 1000 x $1000 \mathrm{~km}$, centrados na área do experimento do Vale do Paraíba. Para a execução da análise proposta, foi necessário escrever a evolução das condições atmosféricas calculadas pelo modelo a cada 15 minutos, já que 
esta era a maior resolução temporal das imagens de satélites (GOES-12). O BRAMS (Longo et al., 2013; Freitas et al., 2009) é um modelo de área limitada com dinâmica não-hidrostática compressível, que permite simulações realísticas do estado atmosférico a partir da assimilação de dados observados e/ ou análises atmosféricas. O modelo conta com estado-da-arte em parametrizações físicas de transferência radiativa, difusão turbulenta na camada limite planetária e microfísica de nuvens, dentre outros. Em termos de microfísica de nuvens, o modelo conta com parametrizações de 1 e 2 momentos (Walko et al., 1995; Meyers et al., 1997). Dois recentes avanços neste sistema trata-se da inclusão do esquema de superfície Joint UK Land Environment Simulator (JULES, Moreira et al., 2013) com ciclo de carbono e parametrização de convecção com ajuste automático à resolução espacial, e com processos de formação e evaporação de chuva dependentes da concentração de CCN (Grell e Freitas, 2013). Outro recente avanço se remete também à dinâmica, com a introdução de um esquema de advecção altamente acurado e monotônico para transporte de escalares (Freitas et al., 2012). A simulação na resolução horizontal de 1 $\mathrm{km}$ utilizou como condições de contorno atmosférico as saídas de uma rodada do modelo BRAMS, configurado com resolução horizontal de $20 \mathrm{~km},(310 \times 210$ pontos $)$, centrada em $59^{\circ} \mathrm{O} ; 3.2^{\circ}$ $\mathrm{S}$, e 48 níveis verticais. Para esta rodada em $20 \mathrm{~km}$, as condições de contorno atmosféricas foram obtidas do modelo global do
CPTEC, versão T382L64 (resolução horizontal 35 km e 64 níveis verticais). A Tabela 1 apresenta mais detalhes sobre os parâmetros de configuração do modelo BRAMS utilizados nas simulações deste estudo.

2. Radiâncias medidas pelos sensores GOES_Imager e $\mathrm{SSMI} / \mathrm{S}$, as quais foram utilizadas na avaliação da convecção resolvida pelo modelo BRAMS. O sensor GOES_Imager está a bordo do satélite GOES-12, que é um satélite de órbita geoestacionária, e realiza medidas em quatro bandas do infravermelho termal $(3.9,6.7,10.2$ e $13.0 \mu \mathrm{m})$ e em uma banda porção visível, resolução espacial de $4 \times 4 \mathrm{~km}$ no ponto sub-satélite e temporal de 15 minutos, além de um sensor de banda larga operando na porção visível, com resolução espacial de 1 x $1 \mathrm{~km}$. O sensor Special Sensor Microwave Imager/ Sounder (SSMIS), a bordo dos satélites Defense Meteorological Satellite Program (DMSP), versões F-17/18, é um radiômetro de micro-ondas passivo linearmente polarizado que realiza medidas em 24 bandas entre as frequências de 19 a $183 \mathrm{GHz}$ em varreduras cônicas com um ângulo de incidência de 53.1 graus. As varreduras são realizadas num ângulo de 144 graus, o que equivale a uma faixa da superfície de aproximadamente $1707 \mathrm{~km}$ de largura.

3. Modelo de transferência radiativa RTTOV, versão 9.3 (Saunders et al., 2010). Foi utilizado para simular as radiâncias dos sensores citados acima.

Tabela 1: Parâmetros utilizados na simulações BRAMS (RAMSIN resumido).

\begin{tabular}{c|c}
\multicolumn{2}{c}{ Atributos de Grade } \\
grade (x,y,z) & $1000,1000,48$ \\
deltaX & $1000 \mathrm{~m}$ \\
deltaY & $1000 \mathrm{~m}$ \\
nudging (tipo) & one-way do próprio modelo \\
pesos relativos para nudging & $1,0.7,0.7,0.5$ \\
parametrização de Cumulus & desligada \\
parametrização de turbulência & $\begin{array}{c}\text { Mellor e Yamada com fechamento } \\
\text { parametrização de superfície }\end{array}$ \\
parognóstico de TKE) \\
adveç̧ão monotonica para escalares & LEAF-3 \\
\hline \multicolumn{1}{c}{ Microfísica } & Freitas et al., 2012 \\
\hline tipo de radiação de onda curta & CARMA \\
tipo de radiação de onda longa & CARMA \\
\hline umidade (nível de complexidade) & 3 \\
espécies microfísicas & 7 \\
nuvens & concentração espcífica \\
cristais de gelo novos (pristine) & concentração prognóstica \\
demais espécies microfísicas & diametro médio específico \\
parâmetros microfísicos & $3 \times 10^{\wedge} 8$ \\
parâmetros de forma para hidrometeoros & 2
\end{tabular}


4. Algoritmo de monitoramento e previsão de curto prazo de sistemas convectivos ForTraCC (Vila et al., 2008). Esta ferramenta foi utilizada para contabilizar o número de células convectivas produzidas pelo BRAMS (através das radiâncias simuladas pelo RTTOV) e aquelas observadas nas imagens GOES reais, bem como a taxa de crescimento, tamanho médio em diferentes estágios do ciclo de vida, duração do ciclo de vida e características do topo dessas nuvens convectivas.

\subsection{Simulações das radiâncias utilizando o modelo de transferência radiativa RTTOV}

Esta seção descreve a metodologia utilizada para simular as radiâncias medidas pelos diversos sensores a bordo de satélites, as quais serviram de base para a avaliação da convecção resolvida pelo BRAMS.

Diversos algoritmos computacionais foram desenvolvidos visando resolver a equação de transferência radiativa para a atmosfera, sendo, consequentemente, denominados "modelos de transferência radiativa". Tais modelos podem ser utilizados para simular o valor das radiâncias que seriam medidas por radiômetros a bordo de satélites para um perfil atmosférico dado, bem como estimar variáveis do perfil atmosférico a partir de radiâncias medidas por satélites, por exemplo, estimar o perfil de temperatura e umidade a partir de dados de um sondador. Este segundo uso dos modelos de transferência radiativa está fora do escopo deste trabalho. Neste estudo, o modelo de transferência radiativa RTTOV foi utilizado visando obter radiâncias para comparação com aquelas observadas a partir de satélites.

Simular as radiâncias dos sensores que operam na porção do infravermelho termal (3.9 a $13 \mu \mathrm{m}$, e.g. satélites GOES, AVHRR, MSG) e dos sensores operantes na porção do microondas (SSMIS, AMSU-A/B, etc) a partir do RTTOV possui certas peculiaridades, pois rotinas distintas são empregadas em cada uma dessas duas classes de sensores. Além das diferenças técnicas da metodologia utilizada pelo RTTOV, uma das varáveis meteorológicas mais importantes para a simulação realística das radiâncias em micro-ondas é a precipitação, em função da forte interação entre as gotas de chuva e a radiação em certos comprimentos de onda da porção do microondas, não sendo de grande importância para as simulações das radiâncias no infravermelho termal.

A partir da versão 9, o modelo de transferência radiativa RTTOV considera o efeito do espalhamento das nuvens na porção do infravermelho termal e a sobreposição de nuvens. Com isso, as radiâncias nessa porção são simuladas de forma mais precisa, porém, é necessário informar a fração de nuvens e a quantidade de água (total líquida e gelo) em cada nível do modelo. Para uma descrição detalhada dos parâmetros necessários e possíveis configurações para execução do modelo de transferência radiativa RTTOV, veja Saunders et al. (1999, 2010). Todas as variáveis utilizadas para as simulações das radiâncias referentes a este estudo são apresentadas na Tabela 2.

Das variáveis apresentadas na Tabela 2, os valores indicados por (*) não foram utilizados diretamente no vetor de estado do RTTOV, serviram apenas como parâmetros auxiliares na determinação do tipo de nuvem presente em cada volume de grade em que existisse nebulosidade. As demais são utilizadas diretamente como parâmetro de entrada no modelo RTTOV. A presença ou não de nebulosidade num dado volume de grade foi definida de acordo com a variável do modelo BRAMS referente ao "total de água condensada". Se essa variável for

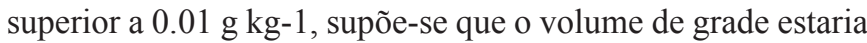
completamente preenchido por nuvens ou completamente sem nuvens caso contrário. Definida a presença ou não de nuvens, a próxima etapa era escolher uma classe de nuvens dentre aquelas possíveis de acordo com o RTTOV. Os tipos de nuvens disponíveis são: Cirrus, Cumulus ou Stratus, sendo as duas últimas classes subdivididas em continental ou oceânica (Figura 1). Estes parâmetros referentes ao tipo de nuvem só se aplicam para as radiâncias do infravermelho termal, pois tal opção não existe para simulações de radiâncias na porção do microondas. A árvore de decisões utilizada para definir um tipo distinto de nuvem para cada nível do modelo é apresentada na Figura 1. Note que a escolha do tipo de nuvem foi baseada na proporção entre as gotículas de água (razão de mistura de

Tabela 2: Variáveis do modelo BRAMS necessárias para simulação das radiâncias do infravermelho e micro-ondas.

\begin{tabular}{c|c} 
Variáveis de superfície & Perfis atmosféricos \\
\hline Temperatura da superfície $[\mathrm{K}]$ & Umidade específica \\
Topografia (máscara continente/oceano) & Temperatura do ar $[\mathrm{K}]$ \\
Temperatura do ar a $2 \mathrm{~m}[\mathrm{~K}]$ & Pressão atmosférica $[\mathrm{K}]$ \\
Pressão à superfície de mar $[\mathrm{hPa}]$ & Razão de mistura de água $[\mathrm{kg} / \mathrm{kg}]$ \\
Umidade específica a $2 \mathrm{~m}$ & Razão de mistura de gelo $[\mathrm{kg} / \mathrm{kg}]$ \\
& Razão de mistura de chuva $[\mathrm{kg} / \mathrm{kg}] * *$ \\
& Total de água condensada $[\mathrm{kg} / \mathrm{kg}] *$
\end{tabular}




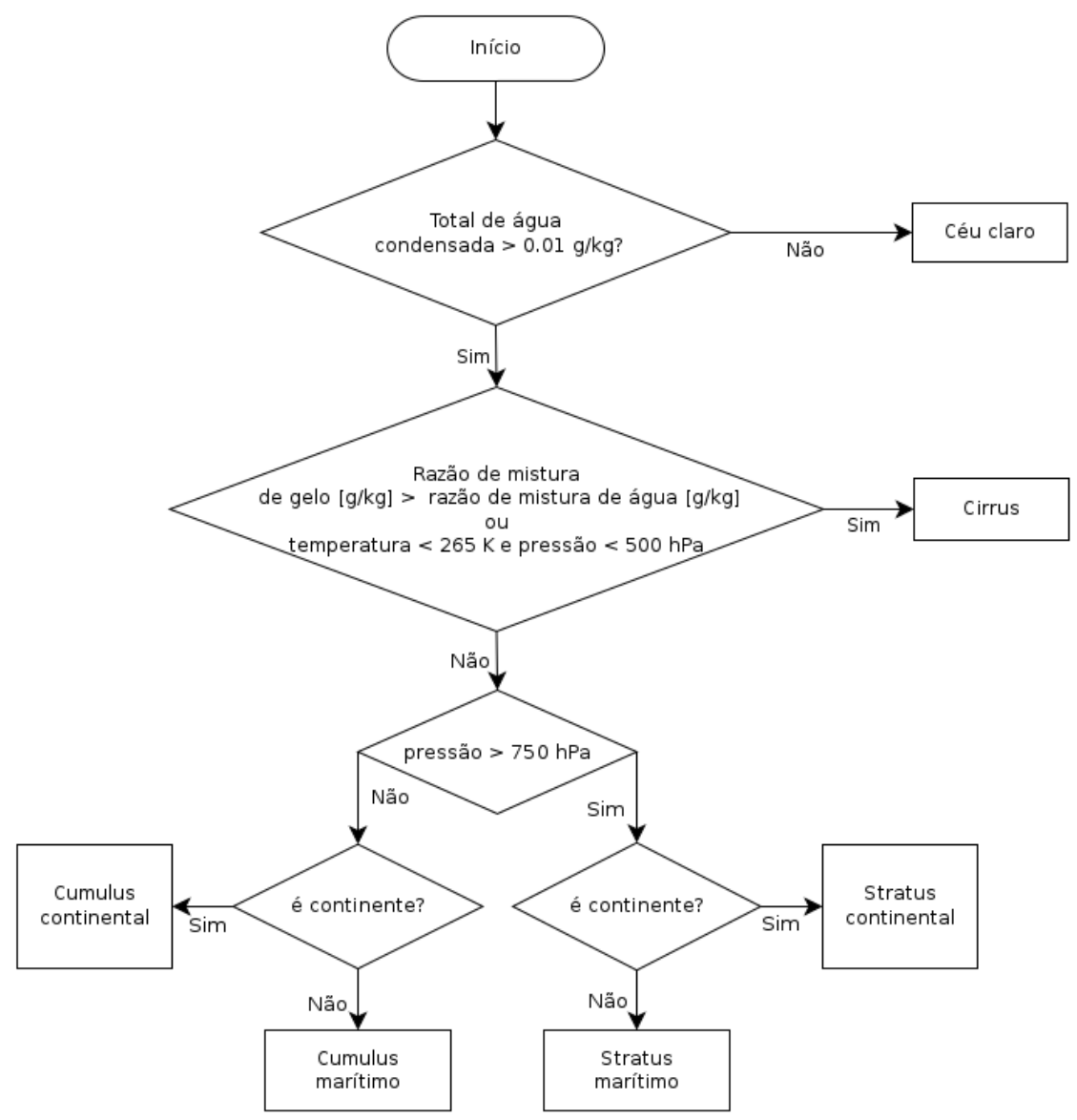

Figura 1: Árvore de decisão para determinação do tipo de nuvem para ser considerado no esquema de multi-espalhamento do RTTOV versão 9.3..

água) e cristais de gelo (razão de mistura de gelo), além de sua altitude e temperatura termodinâmica da camada atmosférica. Para a simulação de radiâncias nas bandas de absorção pelo vapor d'água $(6 \mu \mathrm{m})$ e do infravermelho janela $(11 \mu \mathrm{m})$, as diferenças entre nuvens do tipo Stratus e Cumulus é desprezível, ambas podem ser assumidas como sendo corpos negros. Nuvens Cumulus foram consideradas basicamente em situação de convecção profunda e contribuíram, efetivamente, apenas nos estágios iniciais do desenvolvimento da convecção, quando nuvens Cirrus espessas ainda não haviam se desenvolvido nas camadas superiores. Na presença de nuvens Cirrus associadas ao topo dos sistemas convectivos, a radiação emitida pelas camadas inferiores é completamente absorvida pelas camadas superiores.

Além da escolha do tipo de nuvem, para a simulação das radiâncias utilizando o esquema de múltiplo espalhamento provocado pelas nuvens, é preciso escolher um método de conversão entre o conteúdo de água congelada das nuvens para diâmetro efetivo das gotas (utilizou-se o esquema McFarquar et al. (2003)) e um único tipo de cristal de gelo para cada nível. A descrição das diferentes opções disponíveis pode ser encontrado em Saunders et al. (2010).

\section{ANÁLISE DA CONVECĈ̃̃ A PARTIR DAS RADIÂNCIAS EM CANAIS DA PORÇÃO DO INFRAVERMELHO TERMAL E MICROONDAS}

Esta sessão apresenta a comparação entre a convecção resolvida pelo BRAMS, em termos da distribuição espacial das radiâncias no infravermelho termal e microondas (subseções 4.1 e 4.2) e quão realístico é o ciclo de vida da convecção resolvida pelo BRAMS (subseção 4.3).

A base desta análise se apoia na comparação entre a imagem medida por um satélite em um dado canal com a imagem virtual, no mesmo canal e no mesmo instante, criada pelo BRAMS associado com o modelo de transferência radiativa. Tanto histogramas como as próprias imagens foram utilizadas para essa comparação.

\subsection{Infravermelho}

Para a análise da convecção resolvida pelo BRAMS, foram utilizadas radiâncias na faixa do infravermelho termal dos canais centrados em $6.7 \mu \mathrm{m}$ (banda de absorção do vapor 
d'água), em 3.9 e em $10.2 \mu \mathrm{m}$ (janela atmosférica) do radiômetro a bordo do satélite GOES-12. Estas três bandas permitem avaliar a distribuição da umidade na alta troposfera $(6.7 \mu \mathrm{m})$, as características das nuvens baixas e superfícies continentais $(3.9 \mu \mathrm{m})$ e ciclo de vida da convecção $(10.8 \mu \mathrm{m})$.

Através das imagens do canal em $6.7 \mu \mathrm{m}$, foi verificado que o modelo BRAMS superestima a umidade da alta troposfera, (pico da função peso deste canal está aproximadamente em 450 $\mathrm{hPa}$ ), dado que a $\mathrm{Tb}$ das regiões de céu claro são mais frias nas simulações BRAMS-RTTOV (Figura 2). A ligação entre essa subestimativa da $\mathrm{Tb}$ em $6.7 \mu \mathrm{m}$ e uma superestimativa da temperatura das superfícies continental e oceânica e baixa troposfera podem ser descartadas, pois os valores de Tb das
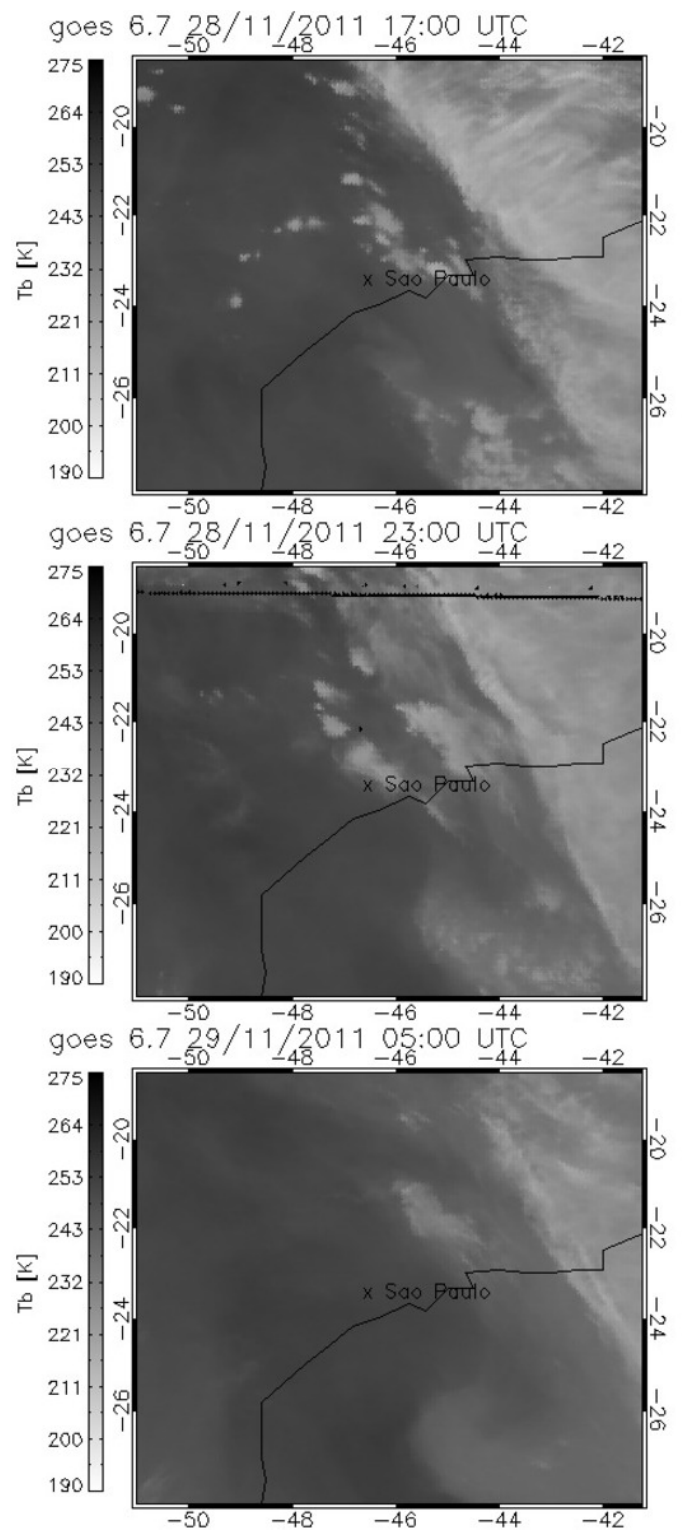

regiões de céu claro nas imagens simuladas para os canais de janela atmosférica são próximos dos observados. A banda do infravermelho próximo centrada em $3.9 \mu$ m é útil para detecção da $\mathrm{Tb}$ dos topos de nuvens baixas e da superfície planetária. Observa-se que os máximos de $\mathrm{Tb}$ dos histogramas referentes ao canal que opera nessa região do infravermelho termal são muito próximo (coluna esquerda da Figura 5), exceto para o histograma do instante inicial das simulações (12:00 UTC). A versão do RTTOV utilizada nesse estudo não considera a radiação solar de onda curta refletida pelas nuvens em $3.9 \mu \mathrm{m}$, portanto, as radiâncias deste canal serão subestimadas durante o período diurno. Essa subestimativa pode ser observada no histograma referente a este canal na Figura 5 para o horário
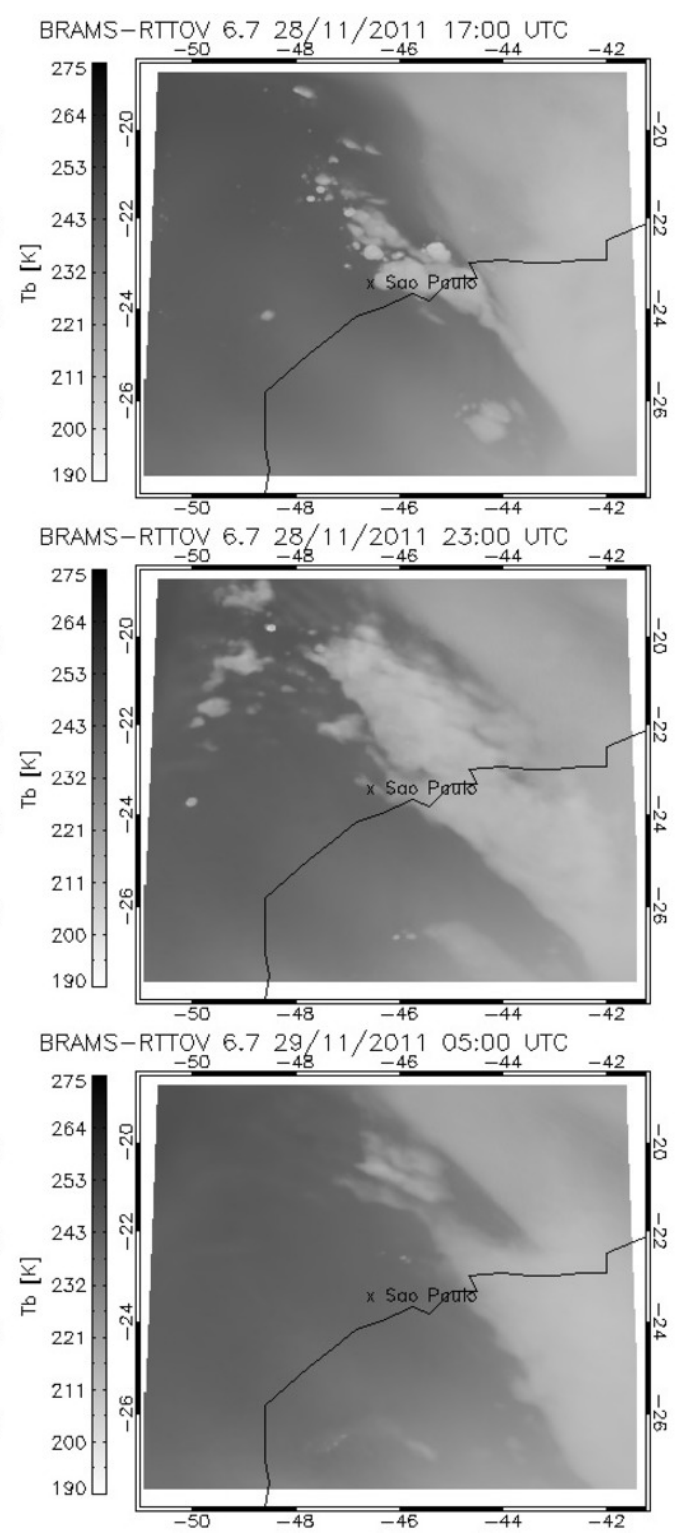

Figura 2: Radiâncias em 6.7 m medidas pelo sensor GOES-Imager (esquerda) e simuladas (direita) para diferentes horários entre 28 e 29 de outubro de 2011. A resolução espacial das imagens reais é de 4 x 4 km, as simulações possuem resolução espacial de 1 x $1 \mathrm{~km}$. 
12:00 UTC. O histograma referente às imagens reais apresenta valor máximo de aproximadamente $310 \mathrm{~K}$, enquanto que para as simulações é próximo de $295 \mathrm{~K}$. Pode ser observado nas imagens do canal em $10.2 \mu \mathrm{m}$, que a quantidade de nuvens baixas ou semi-transparentes é menor nas simulações BRAMS-RTTOV (Figura 3). A banda de nebulosidade que ocupa a porção nordeste do domínio espacial, em que se configurava um padrão da Zona de Convergência do Atlântico Sul (ZCAS), é mais espessa nas simulações, dada a ausência de Cirrus semi-transparentes.

As simulações com o BRAMS tenderam a produzir nuvens convectivas com topos em torno de $14 \mathrm{~km}$ de altura. Os valores mínimos de Tb das imagens do satélite GOES-12, na porção do infravermelho termal, tendem a ser mais quentes
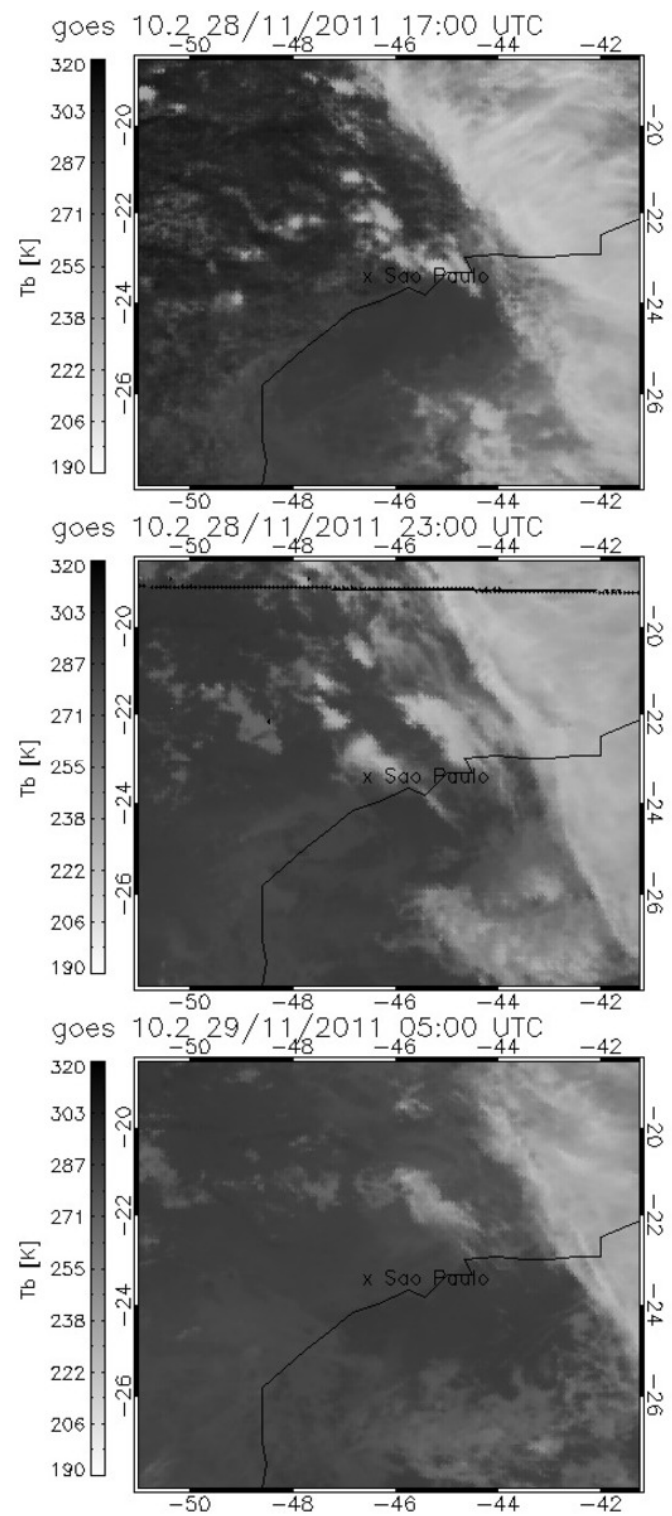

do que nas simulações BRAMS (Figura 4), o que indica que os topos mais altos das nuvens presentes nas imagens reais estão localizados num nível inferior, considerando o lapse-rate da troposfera. Uma vez que a presença de nuvens num volume de grade do modelo depende da existência de água condensada (líquida ou sólida), a altura dos topos das nuvens presentes nas imagens simuladas BRAMS-RTTOV pode ser aferida a partir das concentrações das espécies microfísicas resolvidas pelo BRAMS (Figura 4).

A superestimativa da altura do topo das células convectivas e da concentração de umidade na alta troposfera também são evidenciadas pelos histogramas normalizados de Tb nos canais 3.9, 6.7 e $10.2 \mu \mathrm{m}$, simulados e reais (Figura 5). A
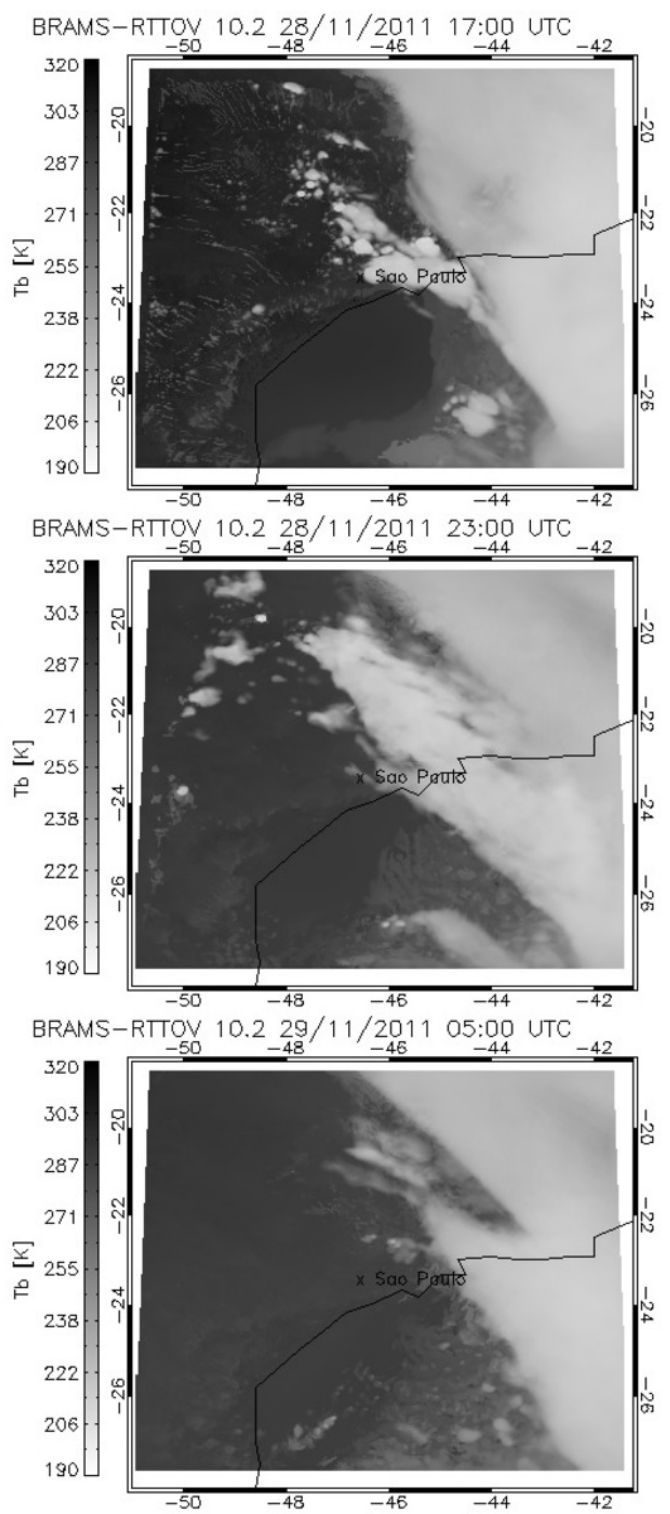

Figura 3: Radiâncias em 10.8 m medidas pelo sensor GOES-Imager (esquerda) e simuladas (direita) para diferentes horários entre 28 e 29 de outubro de 2011. A resolução espacial das imagens reais é de 4 x 4 km, as simulações possuem resolução espacial de 1 x 1 km. 


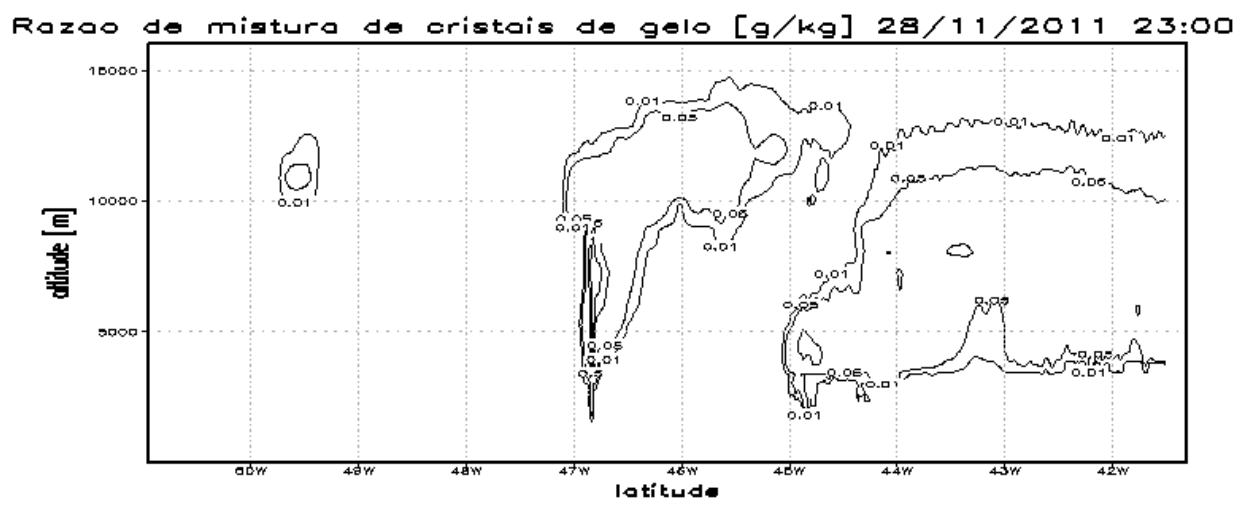

Figura 4: Razão de mistura de cristais de gelo, corte vertical na latitude $22 \mathrm{~S}$ para 23:00 UTC de 28 de novembro de 2011. A existência de nuvens num volume de grade depende da existência de alguma espécie microfísica.
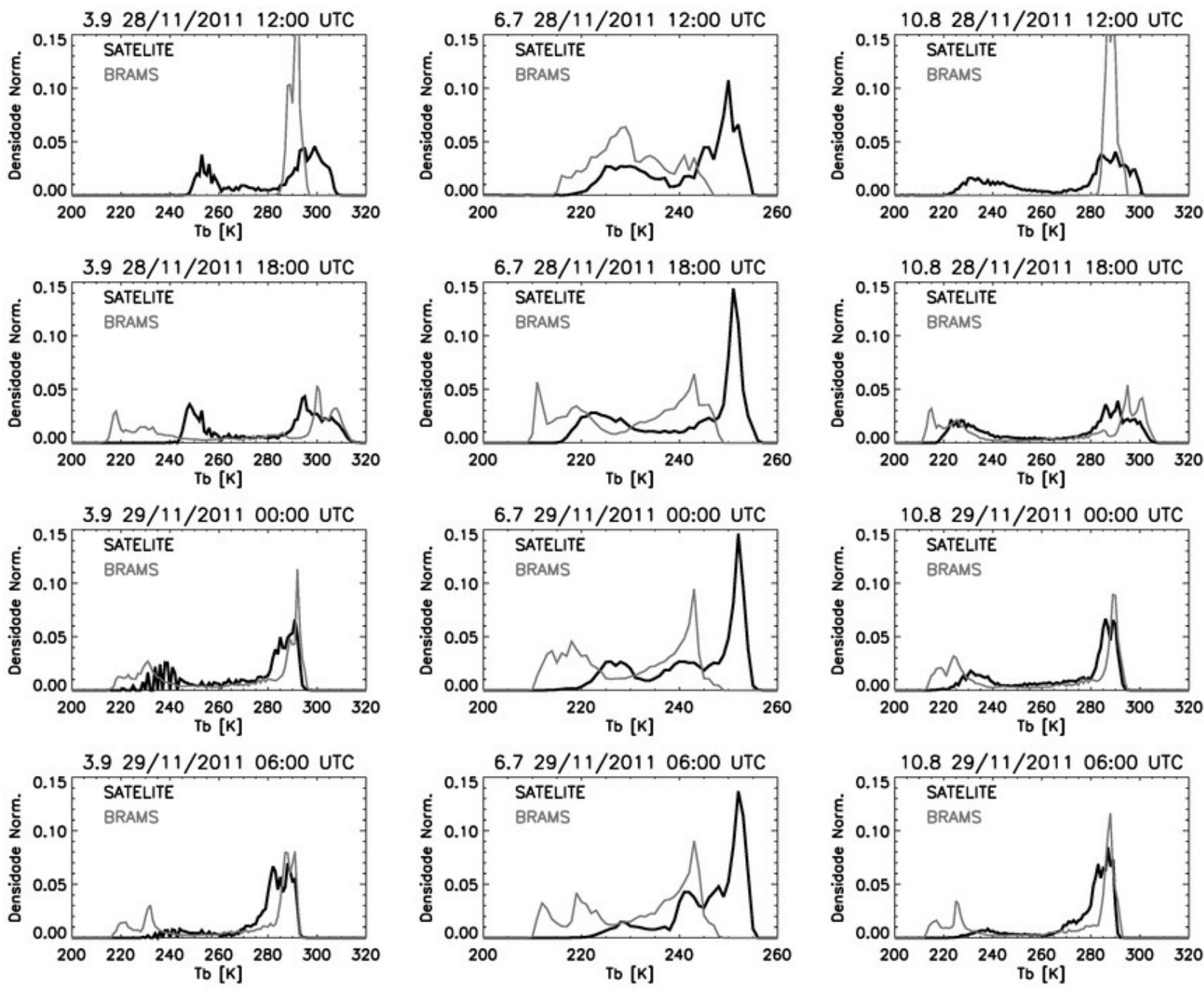

Figura 5: Histogramas de temperatura de brilho para os canais 3.9, 6.7 e $10.8 \mu \mathrm{m}$ do satélite GOES-12 e das simulações BRAMS-RTTOV para horários sinóticos entre 28 e 29 de novembro de 2011.

superestimativa da umidade é indicada pela maior concentração de valores acima de $230 \mathrm{~K}$ para os histogramas referentes às simulações (linhas cinza nos histogramas da coluna central da
Figura 5). Provavelmente, erros na distribuição de umidade ocorridos no processo de inicialização do modelo BRAMS podem ser responsáveis por essa superestimativa de umidade 
em altos níveis. Observa-se também que os valores máximos de $\mathrm{Tb}$ nas simulações ficam em torno de $250 \mathrm{~K}$, geralmente $5 \mathrm{~K}$ mais frios que nas situações reais. $\mathrm{O}$ pico direito dos histogramas de $\mathrm{Tb}$ referente às simulações, nos canais 3.9 e 10.2 $\mu \mathrm{m}$ (colunas esquerda e direita na Figura 5 respectivamente) está relacionado aos valores de $\mathrm{Tb}$ referentes às superfícies continentais e oceânicas e nuvens com topos baixos. A menor largura desse pico nos histogramas referentes às simulações BRAMS (linhas cinzas) indica uma menor frequência de nuvens médias e semi-transparentes. Outro fato que deve ser considerado são as situações em que as nuvens possuem extensão horizontal inferior à resolução espacial do sensor a bordo do satélite (do inglês, sub-grid clouds). Nessas situações, um pixel da imagem pode representar a soma das Tbs dos topos dessas nuvens e da superfície do planeta. Como nas simulações BRAMS esse fato não existe (cada pixel está completamente preenchido por nuvens ou não, seção 2.1), a frequência de $\mathrm{Tb}$ referentes à sub-grid clouds nos histogramas é nula. Para os histogramas referentes ao canal $10.2 \mu \mathrm{m}$, observa-se uma frequência muito maior de $\mathrm{Tb}$ inferiores a $230 \mathrm{~K}$ nas simulações BRAMS-RTTOV em relação à observada nas imagens reais. Isto indica que o modelo produziu nuvens com topos mais altos com maior frequência, o que pode estar relacionado à uma superestimativa dos fluxos verticais nas células convectivas resolvidas pelo modelo.

\subsection{Radiâncias na porção do micro-ondas}

A comparação entre as radiâncias da porção do microondas foi realizada a partir dos histogramas bi-dimensionais calculados a partir de três combinações entre canais do sensor SSMI/S: $91 \mathrm{H}$ vs $91 \mathrm{~V} \mathrm{GHz}, 85 \mathrm{~V}$ vs $37 \mathrm{~V} \mathrm{GHz}$ e 150 vs $91 \mathrm{GHz}$, em que $\mathrm{H}$ e V indicam a polarização dos canais, horizontal e vertical, respectivamente. Os histogramas bi-dimensionais de $\mathrm{Tb}$ (Figura 6) indicam que as simulações não reproduzem a ampla gama de possíveis combinações entre valores de $\mathrm{Tb}$, que são observadas nas imagens reais. Por exemplo, os valores da $\mathrm{Tb}$ em $150 \mathrm{GHz}$, que são úteis para estimar o caminho óptico do gelo, são superiores a $240 \mathrm{~K}$, indicam ausência de camadas espessas com alta concentração de cristais de gelo uma vez que os valores simulados concentram-se em torno de $270 \mathrm{~K}$ (Figura 6). Os valores das radiâncias observadas nos canais espectrais da porção do microondas, utilizadas para esta análise, se devem, principalmente, à emissividade da superfície, em que os valores mínimos estão relacionados ao espalhamento da radiação por partículas de gelo, gotas relativamente grandes ou precipitação. Devido à convecção ser produzida pelo modelo sob a forma de torres compactas e isoladas, as espécies microfísicas associadas à precipitação, consequentemente, são concentradas em pequenas regiões do domínio espacial. As simulações BRAMS-RTTOV foram degradadas para a mesma resolução espacial das imagens de satélites ( 1 x $1 \mathrm{~km}$ para $\sim 12.5$ x 12.5 $\mathrm{km})$ para a confecção desses histogramas bi-dimensionais. Tal degradação foi feita a partir de uma média aritmética simples. Logo, regiões em que a concentração de cristais de gelo era suficiente para influenciar no valor das radiâncias em 150 ou 91 $\mathrm{GHz}$, mas que se concentravam numa área horizontal inferior a $12.5 \mathrm{~km}^{2}$, foram consideradas juntamente com regiões de céu claro circunvizinhas, resultando num valor de $\mathrm{Tb}$ superior. Portanto, as radiâncias referentes à radiação emitida pelas superfícies continental e oceânica predominam em percentual de área horizontal. Isso explica a alta frequência de valores de $\mathrm{Tb}$ próximos aos máximos observados, nos histogramas referentes às radiâncias simuladas.

Em geral, a amplitude dos valores máximos e mínimos de $\mathrm{Tb}$ é maior nas simulações. Visto que os valores de $\mathrm{Tb}$ das simulações dos canais do infravermelho janela são muito próximos daqueles observados nas medidas reais, apresentando valores superiores em poucos graus, esta superestimativa dos máximos de Tb na porção do micro-ondas já era esperada. Para as simulações, observa-se uma relação quase simétrica entre as radiâncias observadas em ambos os canais. Este comportamento deve estar relacionado à presença de um único tipo de cristal de gelo, que é assumida durante os cálculos de transferência radiativa pelo modelo RTTOV. O mesmo é observado para os histogramas referentes às regiões continentais para as demais combinações entre canais.

\subsection{ForTraCC}

A ferramenta ForTraCC (Vila et al, 2008) utiliza apenas imagens da janela atmosférica em torno de $11 \mu \mathrm{m}$, apenas as radiâncias em $10.8 \mu \mathrm{m}$ do satélite GOES-12 foram necessárias. A resolução temporal das imagens (reais e simuladas) utilizadas foi de 15 minutos, o valor limítrofe da área horizontal mínima e mínima $\mathrm{Tb}$ para a definição dos sistemas convectivos a serem rastreados foi de $1200 \mathrm{~km}^{2}$ e $265 \mathrm{~K}$.

Dada a alta resolução horizontal do modelo (1 x $1 \mathrm{~km}), \mathrm{o}$ nível de detalhamento das células convectivas é superior àquele das imagens reais, permitindo distinguir células convectivas pequenas muito próximas. Nuvens vizinhas cujo espaçamento horizontal entre elas for inferior à resolução espacial de um dado sensor, serão detectadas como sendo uma única nuvem por este sensor. Este fato explica porque o tamanho médio das classes de sistemas convectivos identificados nas imagens reais é maior na Figura 7. O curto período de tempo das simulações (36 horas) e domínio espacial relativamente pequeno (1000 x $1000 \mathrm{~km}$ ) impossibilitou a análise de sistema convectivos de longa duração, uma vez que tais sistemas são, geralmente, 

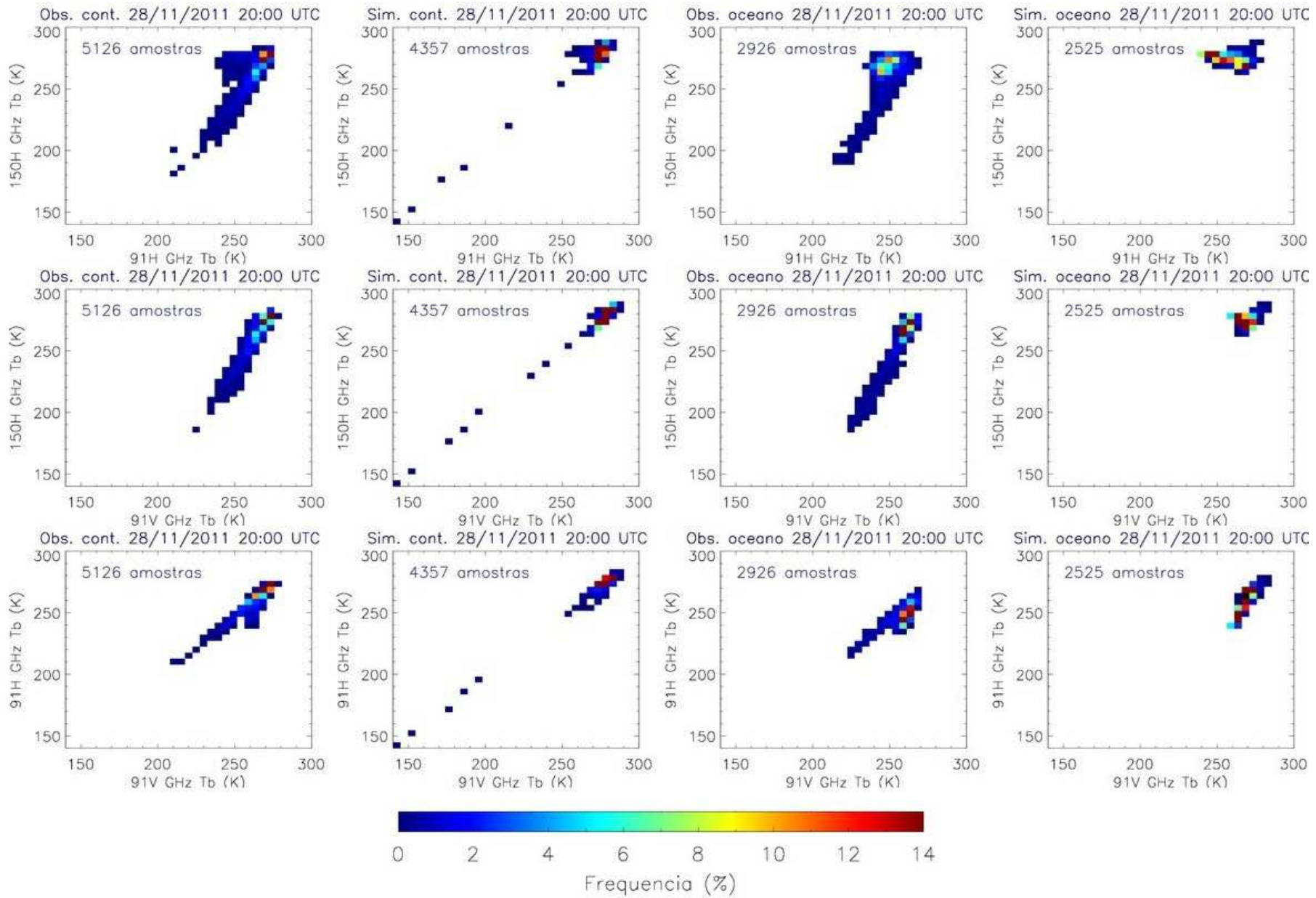

Figura 6: Histogramas bi-dimensionais para várias combinações entre diferentes canais do sensor SSMIS-S referentes às 20:00 UTC do dia 28 de novembro de 2011.

extensos horizontalmente. As simulações utilizadas nesse estudo permitiram avaliar a atividade convectiva de sistemas ou células de até $2000 \mathrm{~km}^{2}$ de área horizontal.

Para a confecção dos gráficos na Figura 7, apenas sistemas convectivos que não se fundiram ou se dividiram foram considerados nessa análise, e os sistemas foram agrupados de acordo com a duração do seu ciclo de vida, de forma similar ao que foi feito por Negri et al. (2014). Note que o tamanho médio das células convectivas no modelo é sempre muito inferior ao tamanho observado na realidade. Diferentemente de Negri et al. (2014), as imagens do canal $10.2 \mu \mathrm{m}$ simuladas a partir dos perfis atmosféricos do BRAMS não foram degradadas para a mesma resolução das imagens do satélite GOES-12. Degradando a resolução espacial das simulações para $4 \times 4 \mathrm{~km}$, ter-se-ia imagens com apenas $250 \times 250$ pontos, o que não proporcionaria uma análise estatisticamente significante. Para a comparação com as imagens GOES-12, um cenário ideal compreenderia um domínio espacial da ordem de 2000 x 1500 pontos e com resolução de ponto de grade de 1,5 ou $2 \mathrm{~km}$.
Apesar dessas limitações, a análise confirma a tendência do modelo em produzir células convectivas isoladas e subestima o desenvolvimento de nuvens Cirrus associadas à divergência do vento no topo dos sistemas convectivos. Em situações em que uma ou mais células convectivas próximas estariam com seus topos conectados por uma camada de Cirrus, o ForTraCC identificaria como um sistema maior.

Em relação à taxa de crescimento, observa-se uma grande diferença para sistemas com duração máxima de 1 hora (linha verde claro). Os sistemas dessa classe, presentes nas simulações BRAMS, apresentam taxas de crescimento (e decrescimento) muito superiores ao que é observado na situação real. Para as demais classes de sistemas, observa-se que a taxa de crescimento é sempre superior nas simulações BRAMS, porém após atingirem o tamanho máximo e iniciarem a fase de dissipação, a taxa de decrescimento é similar àquela observada para os sistemas presentes nas imagens reais. As taxas de decrescimento dos sistemas, nas simulações e nas imagens reais, com duração de até 4 horas ou superior a 4 horas são similares. Esse fato 

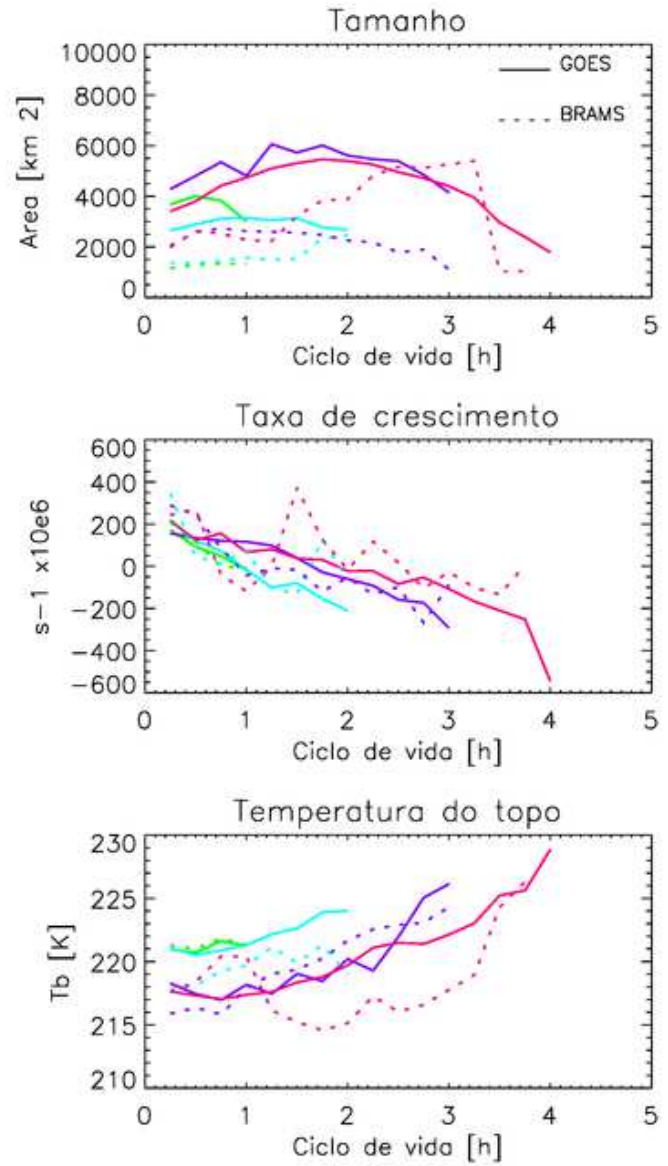

Figura 7: Tamanho médio, taxa de crescimento e temperatura de brilho média dos sistemas convectivos observados nas simulações BRAMS/RTTOV (linhas pontilhadas) e nas imagens GOES reais (linhas contínuas) para 28 a 30 de novembro de 2011 para sistemas com duração de até 1 (verde), 2 (azul claro), 3 (roxo) ou 4 (rosa) horas.

indica que os fluxos verticais de massa simulados pelo BRAMS são muito intensos, possivelmente o controle do entranhamento poderia reduzir esse efeito.

Ao analisar a temperatura de brilho média do topo dos sistemas convectivos, verifica-se que os topos dos sistemas de curta duração (até 2 horas), presentes nas simulações, tendem a atingir altitudes mais baixas ( $\mathrm{Tb}$ mais quentes) em relação aos sistemas presentes nas imagens reais. Para os demais sistemas (simulações e imagens reais), os valores são similares.

Esta análise indicou algumas deficiências do modelo BRAMS, bem como, pontos em que ele é capaz de reproduzir as características observadas na situação real. Contudo, o domínio e a resolução espacial das simulações não favorece esta metodologia de análise. A impossibilidade de degradar espacialmente as simulações BRAMS/RTTOV, de forma a reproduzir a mesma resolução das imagens GOES reais, comprometeu a análise do ciclo de vida dos sistemas convectivos associados a células convectivas pequenas e muito próximas.

\section{CONCLUSÕES}

Este trabalho explorou o uso da metodologia apresentada por Negri et al. (2014) aplicando-a à uma série de simulações em escala de nuvens realizada no CPTEC/INPE, como parte do projeto CHUVA, e buscou aprimorar a metodologia ao utilizar imagens da porção do micro-onda possibilitando avaliar as características da precipitação e distribuição de hidrometeoros resolvidas pelo BRAMS.

A metodologia foi útil, apontando as deficiências no esquema de resolução explicita de nuvens, o que poderá auxiliar a melhoria desse tipo de simulação numérica que é realizada pelo CPTEC/INPE.

Os resultados indicam que as simulações para outros casos selecionados para estudo, referentes aos demais experimentos de campo do projeto CHUVA, podem ser avaliadas seguindo a metodologia apresentada neste texto. Dessa forma, ter-se-á um conjunto mais extenso de situações com presença de sistemas convectivos que permitirá realizar uma comparação entre os perfis microfísicos calculados pelo modelo BRAMS e os perfis médios observados durante os respectivos experimentos de campo do projeto CHUVA. Esta comparação poderá indicar a acurácia do modelo em representar as distribuições verticais dos hidrometeoros presentes nas torres de convecção profunda que ocorrem nas diferentes regiões do território brasileiro, foco do projeto CHUVA.

Para as simulações referentes ao experimento do Vale do Paraíba, as quais foram avaliadas neste estudo, a metodologia indicou que o modelo BRAMS:

Não produziu nuvens médias em quantidade similar àquela observada nas imagens reais.

- A distribuição da umidade nos altos níveis (acima de 400 $\mathrm{hPa}$ ) é superestimada e este erro se elevava gradualmente a medida que a simulação numérica evolui.

- A localização das células convectivas (e complexos) está próxima à realidade, o modelo produziu complexo convectivos extensos, mas tende a produzir células pequenas (dezenas de quilômetros), mesmo considerando as diferenças nas resoluções espaciais (1x1 km versus $4 \times 4 \mathrm{~km})$.

- Tende a produzir nuvens Cirrus espessas em excesso em algumas regiões do domínio espacial, não sendo fisicamente. Como este fato ocorre sistematicamente em algumas regiões, é possível descartar tais regiões facilmente de acordo com o uso pretendido das simulações. 
- A precipitação resolvida se resumiu a situações isoladas de algumas dezenas de quilômetros em extensão horizontal.

- Tende a concentrar a precipitação em pequenas áreas. Numa imagem típica do micro-ondas, é comum identificar sistemas precipitantes com extensões horizontais de centenas de quilômetros, o que não foi observado na simulações.

\section{AGRADECIMENTOS}

Agradecemos ao Instituto Nacional de Pesquisas Espaciais (INPE) por fornecer a infraestrutura para o desenvolvimento do projeto de pós-doutorado do qual este trabalho é derivado e ao Conselho Nacional de Desenvolvimento Científico e Tecnológico (CNPq) por fornecer a bolsa de pósdoutorado, processo $n^{\circ}$ 501324/2012-7.

\section{REFERÊNCIAS BIBLIOGRÁFICAS}

CHABOUREAU, J. P.; CAMMAS, J. P.; MASCART, P.; PINTY, J.P.; CLAUD, C.; ROCA, R.; MORCRETE, J.J. Evaluation of a cloud system life-cycle simulated by the Meso-NH model during FASTEX using METEOSAT radiances and TOVS-3I cloud retrievals Part I. Quarterly Journal of the Royal Meteorological Society, v. 126, 566, p. 1735-1750, 2000.

DONE, J.; DAVIS, C. A., WEISMAN M. the next generation of NWP: Explicit forecasts of convection using the weather research and forecasting (WRF) model. Atmospheric Sciency Letters, v. 5, p. 110-117, 2004.

FREITAS, S. R.; LONGO, K. M.; SILVA DIAS, M. A. F., et al. The Coupled Aerosol and Tracer Transport model to the Brazilian developments on the Regional Atmospheric Modeling System (CATT-BRAMS) - Part 1: Model description and evaluation, Atmospheric Chemistry Physics, v. 9, p. 2843-2861, 2009.

FREITAS, S. R.; RODRIGUES, L. F.; LONGO, K. M.; PANETTA, J. Impact of a monotonic advection scheme with low numerical diffusion on transport modeling of emissions from biomass burning, Journal of Advances in Modeling Earth Systems, 4, M01001, doi:10.1029/2011MS000084, 2012.

GRELL, G. A.; FREITAS, S. R. A scale and aerosol aware stochastic convective parameterization for weather and air quality modeling, Atmospheric Chemistry and Physics, v. 13, p. 23845, 2013.

LEAN, H. W.; CLARK, P. A.; DIXO, M.; ROBERTS. N. M., FITCH A., FORBES R., HALLIWELL C. Characteristics of high-resolution versions of the Met Office Unified Model for forecasting convection over the United Kingdom. Monthly Weather Review, v. 136, p. 3408-3434, 2008.

LONGO, K. M. et al. The chemistry CATT BRAMS model (CCATT-BRAMS 4.5): a regional atmospheric model system for integrated air quality and weather forecasting and research, Geoscientific Model Development, v. 6, p. 1389, 2013.

MACHADO, L. A. T. et al. The CHUVA Project - how does convection vary across Brazil? Bulletin Of American Meteorology Society (2014) (in press).

MCFARQUHAR, G. M.; IACOBELLIS, S.; SOMERVILLE, R. C. J. SCM simulations of tropical ice clouds using observationally based parameterizations of microphysics. Journal of Climate, v. 16, p. 1643-1664, 2003.

MOREIRA, D. S. et al. Coupling between the JULES landsurface scheme and the CCATT-BRAMS atmospheric chemistry model: applications to numerical weather forecasting and the $\mathrm{CO} 2$ budget in South America, Geoscientific Model Development, v. 6, p. 1243, 2013.

MEYERS, M. P. et al. New RAMS cloud microphysics parameterization. Part II: The two-moment scheme. Atmospheric Research, v. 45, n. 1, p. 3-39, 1997.

NEGRI, R. G.; MACHADO L. A. T.; ENGLISH, S.; FORSYTHE, M. Combining Cloud Resolving Model with Satellite for Cloud Process Model Simulation Validation. Journal of Applied Meteorology and Climatology, v. 53, p. 521-533, 2014.

PLOSHAY, J. J.; LAU, N. C. Simulation of the diurnal cycle in the tropical rainfall and circulation during boreal summer with a high-resolution GCM. Monthly Weather Review , v. 138, p. 3434-3453, 2010.

ROMERO, R.; DOSWELL, C. A.; RIOSALIDO, R. Observations and fine-grid simulations of a convective outbreak in northeastern Spain: Importance of diurnal forcing and convective cold pools. Monthly Weather Review , v. 129, p. 21572182, 2001.

SATO, T.; MIURA, H.; SATOH, M.; TAKAYABU, Y. N.; WANG, Y. Diurnal cycle of precipitation in the tropics simulated in a global cloud-resolving model. Journal of Climate, v. 22, p. 4809-4826, 2009.

SAUNDERS, R. W.; MATRICARDI, M.; BRUNEL, P. An Improved Fast Radiative Transfer Model for Assimilation of Satellite Radiance Observations. Quarterly Journal of the Royal Meteorological Society, 125, 1407-1425, 1999.

SAUNDERS R.W.; MATRICARDI, M.; GEER, A. RTTOV-9 Users Guide version 1.7., $2010 \mathrm{http}: / /$ research.metoffice. gov.uk/research/interproj/nwpsaf/rtm/rttov9_files/users_ guide_9_v1.7.pdf, acessado em 24 de Maio de 2012.

SPEER M. S., LESLIE L. M. The prediction of two cases of severe convection: implications for forecast guidance. 
Meteorology and Atmospheric Physics, v. 80, p. 1-4, 2002.

VILA, D. A., MACHADO, L. A. T., LAURENT, H., Velasco, I. Forecast and Tracking the Evolution of Cloud Clusters (ForTraCC) Using Satellite Infrared Imagery: Methodology and Validation. Weather and Forecasting, v. 23, p. 233, 2008 .
WALKO, R. L., COTTON, W. R., MEYERS, M. P. AND HARRINGTON, J. Y. New RAMS cloud microphysics parameterization. Part I: the single-moment scheme, Atmospheric Research, v. 38, p. 29-62, 1995. 